\title{
Advanced Possibilities of Fast Quality Check in Wire Production Processes by Means of Fast Microstructure Analysis
}

\section{Anja Oswald ${ }^{1, a}{ }^{*}$, Rosita Schmidtchen ${ }^{1, b}$, Daniel Šimek ${ }^{2,3, c}$, David Rafaja ${ }^{3, d}$, Rudolf Kawalla ${ }^{1, \mathrm{e}}$, Gunter Lehmann ${ }^{1}$}

${ }^{1} \mathrm{TU}$ Bergakademie Freiberg, Institute of Metal Forming, Bernhard-von-Cotta-Str. 4, 09599 Freiberg, Germany

${ }^{2}$ Czech Academy of Sciences, Institute of Physics, Na Slovance 2, 18221 Praha 8, Czech Republic

${ }^{3}$ TU Bergakademie Freiberg, Institute of Materials Science, Gustav-Zeuner-Str. 5, 09599 Freiberg, Germany

\author{
${ }^{a} A n j a . O s w a l d @ i m f . t u-f r e i b e r g . d e,{ }^{b}$ Rosita.Schmidtchen@imf.tu-freiberg.de, \\ csimek@fzu.cz, drafaja@iww.tu-freiberg.de, ${ }^{\mathrm{e} R u d o l f . K a w a l l a @ i m f . t u-f r e i b e r g . d e ~}$
}

Keywords: carbon steel, spring steel, pearlite, microstructure analysis, hot rolling, wire drawing, Xray diffraction, mechanical testing, quality assurance, microstrain, anisotropy

\begin{abstract}
A new method for a fast analysis of heavily deformed, multicomponent ferritic/pearlitic steels microstructure based on XRD measurements had been developed. Its practical application has been examined and proven during wire rod production of a high-strength eutectoid non-alloyed steel grade containing 0.81 weight percent carbon. For individual technological conditions, the lattice strains and their anisotropy were analysed quantitatively by means of fast X-ray diffraction measurements and correlated with the results of comprehensive mechanical testing. Obtained relationships between the microstructure characteristics and mechanical properties were described using physically based models and used to establish a material specific database for prediction of the mechanical properties from X-ray diffraction data. Depending on the deformation state different parameters have to be applied for the material's macroscopic properties prediction. Additionally, the fast microstructure analysis can provide more detailed information in the case of deviations from the as-required material's properties due to technological aberrations.
\end{abstract}

\section{Introduction}

The most often high-end applications of high-strength cold drawn non-alloyed spring steels are associated with strict quality criteria, in particular concerning the chemical composition, the geometric accuracy, the surface quality and the microstructural features [1]. To a great amount, the microstructure influences the mechanical properties of the steels and is strongly influenced by technological factors. During the production of steel rods and wires, the quality control is usually performed by mechanical testing, such as tensile tests or hardness measurements. For more detailed information, the metallographic preparation and examination of the samples is necessary. The related methods and procedures are often very time consuming, and their evaluation and interpretation can easily be affected by subjective impressions. Therefore, the investigations reported below focused at the development of a new method, which should be able to provide reliable results concerning the wires' quality very quickly by almost completely automated measurements and evaluation. Furthermore, the method should be practicable without great effort by non-specialized personnel, too.

Within the scope of a progressive research cooperation, a new method of Fast Microstructure Analysis (FMA) of heavily deformed non-alloyed steel with ferritic-pearlitic microstructure based on X-ray diffraction (XRD) has been developed recently. It is generally known that the microstructure characteristics like grain and substructure refinement or residual stresses observed at atomic scale are directly related to the steel's mechanical properties and to its technological behaviour. This information can be made accessible by means of the FMA. The method applied 
during the present investigations had first been developed and tested under laboratory conditions on a medium carbon steel C45 with ferritic-pearlitic microstructure in as hot and cold deformed condition as well [2] and later on eutectoid C80, too [3,4]. The method was now transferred to and tested in an industrial scale application, namely in the production of high-strength steel wire for spring fabrication.

\section{Experimental procedure}

Due to the material specific microstructure characteristics of the examined steel grade, the microstructure models as well as the microstructure database initially created for the non-alloyed medium carbon steel $\mathrm{C} 45$ had to be enhanced and expanded. Therefore, a number of reference data had to be created experimentally referring to the technological boundary conditions that may occur during wire production and the microstructural and mechanical properties achievable during processing via hot rolling and wire drawing. The experimental data for filling the database and adapting the corresponding microstructure models have all been generated from material of the same steel batch, smelted and cast in an industrial scale process. The chemical composition of the examined melt is given in Table 1.

Tab. 1. Chemical composition of the examined eutectoid steel grade C80 (D2)

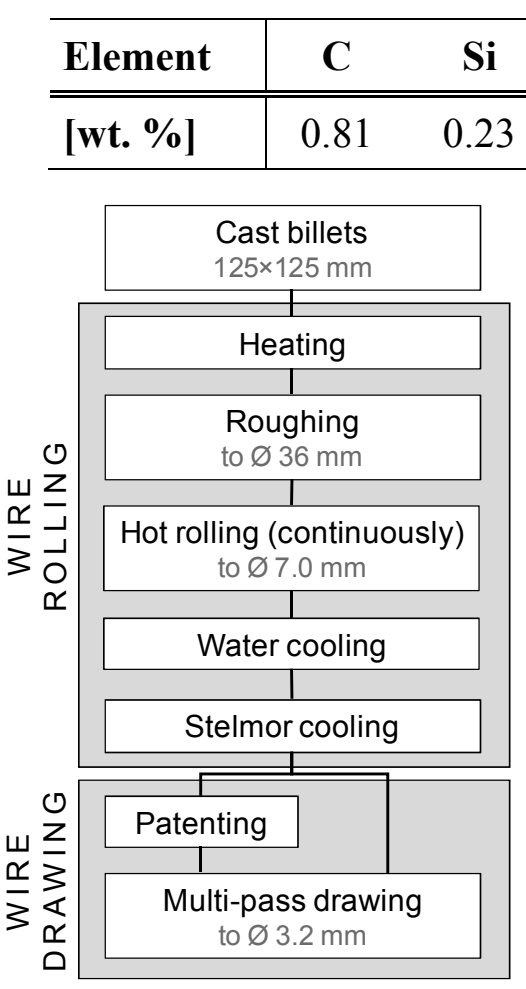

Fig. 1. Technological procedure for the experimental examination of spring steel wire production applied to the samples R1 to R4

At first, the typical industrial process chain was analyzed during real production starting at the wire rod production by hot rolling, optional heat treatment and subsequent cold deformation by wire drawing. To ensure comparability to the practical application the examinations were completely performed in industrial scale. Thereby, the material was 'tracked' continuously during the whole processing and samples were taken during the processes according to the particular possibilities and were analysed by mechanical testing. The characterization of the samples technology-related microstructure was performed by means of FMA as well as by conventional microstructure analytical methods (optical microscopy (OM), scanning electron microscopy (SEM) and transmission electron microscopy (TEM)) to get comprehensive information for the microstructure modelling and the basic data needed therefor. An overview of the technological sequence for the industrial scale experiments can be seen in Figure 1. 
Hot Rolling. The experimental analysis of wire rod production process was performed on an industrial rolling plant under conditions of real production. Four continuously cast billets with a $125 \times 125 \mathrm{~mm}^{2}$ cross section (in the following referred to as R1, R2, R3 and R4) of a unit weight of approx. 1.5 tons were heated in a continuous furnace to $1150{ }^{\circ} \mathrm{C}$ and hot rolled in an oval/round pass sequence to a final dimension of $\varnothing 7.0 \mathrm{~mm}$. The rolling was performed continuously in one heat and at rolling speeds of up to $75 \mathrm{~m} / \mathrm{s}$. To achieve different microstructures or pearlite modifications, respectively, the cooling conditions (and therefore the conditions for static recrystallisation and $\gamma / \alpha$-transformation) have been modified during cooling of wire rods after hot rolling. The wire rods R1 and R4 underwent a standard cooling procedure, including a water precooling before passing the loop layer and a subsequent Stelmor cooling on a layer belt. For the R2 wire rod a malfunction in the air cooling unit was simulated with cooling efficiency reduced to only $25 \%$. The R3 sample experienced a loss of water cooling of $15 \%$. The R4 wire cooled to room temperature at standard conditions after hot rolling was subjected to an additional heat treatment prior to drawing to attain an especially fine pearlite structure which would not have been achieved by standard cooling technology. This patenting operation included austenitization at temperatures slightly above $A_{\mathrm{C} 3}$ and subsequent isothermal transformation in a lead bath.

Wire Drawing. Following the technological chain, the further processing of the as-rolled and differently cooled industrial samples R1 to 4 with $\varnothing 7.0 \mathrm{~mm}$ were then subjected to industrial wire drawing. The drawing experiments were performed using a multi-pass industrial drawing machine. For all wires R1 to R4 the same 9-step drawing pass sequence and equal drawing conditions concerning lubrication, drawing dies, drawing speeds were applied to ensure comparability of the samples. The wire heating during cold deformation was measured on the surface by non-contact method via infrared thermometer. Samples for microstructure analysis and mechanical testing had been taken after the second, fourth, sixth and ninth drawing pass. All the examined wires have been drawn to final dimension of $\varnothing 3.2 \mathrm{~mm}$. Therefore, the effective total (logarithmic) drawing strain was about $\varphi_{t}=1.57$ after the last drawing pass.

Microstructure analyses. The XRD measurements were carried out by using a URD 6 diffractometer from Freiberger Praezisionsmechanik, which was equipped by sealed X-ray tube with cobalt anode and a position sensitive linear detector to accelerate the measurements. The SEM observations were done using LEO 1530 from Carl Zeiss, the TEM experiments using the JEM 2200 FS from Jeol.

\section{Results and Discussion}

Method of Fast Microstructure Analysis. A comprehensive description of the FMA method and the physically based microstructure models have already been given in [2] and [5]. Therefore, in the following only the key points will be summarized:

As mentioned before FMA is based on X-ray diffraction (XRD) which has several advantages accounting for its special suitability for this application. The sample preparation is easy and involves minor effort. It only consists of cutting and slightly sanding to prevent effects on the local stress conditions. A metallographic preparation is not necessary. The measurement itself can be performed in relatively short time with sufficient precision and sensing depth and can also be done automated, as well as the evaluation of the data. An additional benefit is the fact that the effects which can be measured by means of the XRD are directly related to the steel's mechanical properties.

The evaluation of the XRD patterns is based on the analysis of the diffraction line profiles. Thereby, only selected diffraction lines are detected leading to a significant speed up of the procedure compared to complete diffraction line measurements. In the case of pearlitic steels typically those for the $h k l$ indices 110, 200, 211 and 220 of the bcc ferritic phase are determined. The analysis of the diffraction lines regarding peak positions, integrated intensities, profile breadths and line shapes allows to draw conclusions on residual stresses, microstructure defects and related microstrains and also about the preferred orientation of crystallites (texture). For this reason, the 
peaks are fitted by an empirical Pearson VII function [6] to get the maximum intensities $I_{\max }$, the line positions in the units of the reciprocal space $(2 \sin \theta / \lambda)=1 / d$ (where $d$ accounts for the mean interplanar distance, $\lambda$ for the wavelength of the X-rays and $2 \theta$ for the diffraction angle), the full widths at half maximum (FWHM) $w$ and the shape parameters $m$ for all XRD lines $h k l$. From these four fit parameters, the appropriate microstructure parameters can be calculated [5], which were found to be characteristic for the examined deformation state and processing conditions, as described below.

FMA after hot rolling. All samples prepared by hot rolling during industrial processing exhibited a fully pearlitic macroscopic microstructure. Depending on the accelerated cooling conditions, the morphology of pearlite was found to differ concerning colony size and the mean interlamellar distance $I L D$ in particular. The coarsest structure was observed for sample R2 transformed under reduced air cooling efficiency showing a very similar visual appearance during $\mathrm{OM}$ and SEM examinations. The reduced water cooling performed at sample R3 hardly effected the pearlite macrostructure in comparison to sample R1 prepared under 'standard' cooling conditions. The patenting operation performed on sample R4, however, led to a distinct refinement of the lamellar structure as can be seen in the SEM micrographs in Figure 2. The macroscopic material strength and ductility properties (yield strength $Y S$, ultimate tensile strength UTS, elongation at break $A$ and area reduction at break $Z$ ) of the as-rolled wires were examined in tensile tests and are shown in Figure 3. As expected, referring to their lamellar structure characteristics, sample R2 showed the lowest strength and ductility values, while the patented wire R4 reached rather high values of these properties.
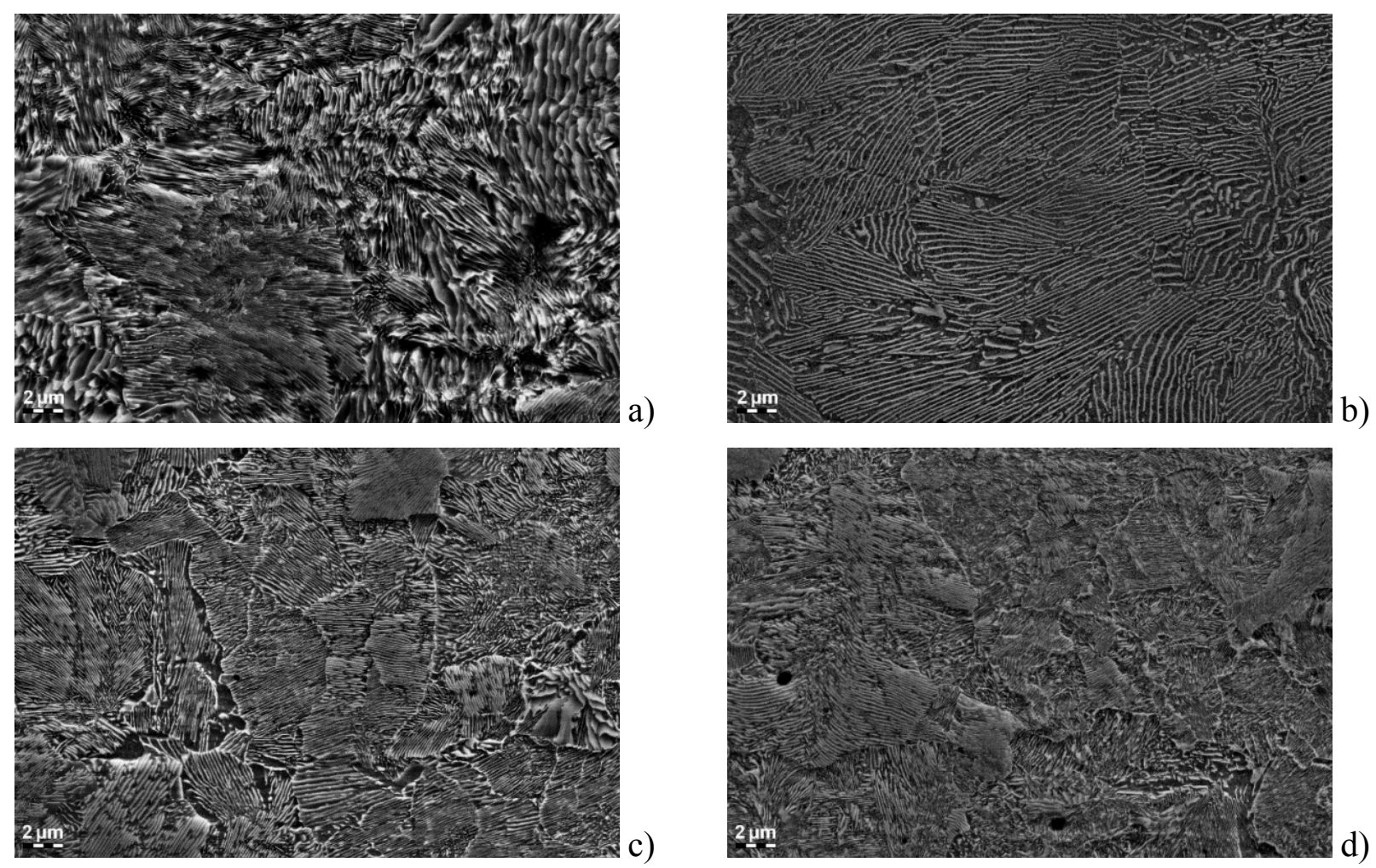

Fig. 2. SEM micrographs of as-rolled wire samples R1 (a), R2 (b) and R3 (c) exposed to varying cooling conditions and of the as-patented sample R4 (d), all showing fully pearlitic microstructures with different modifications of lamellar structure 


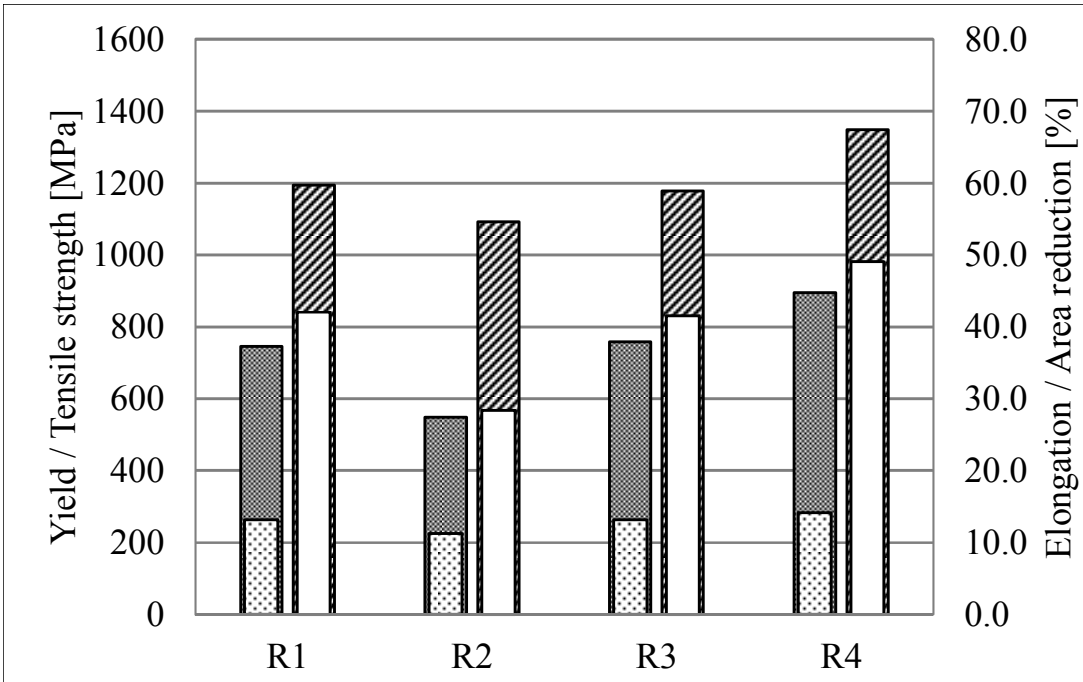

圆 Yield strength $\square$ Tensile strength $\boxminus$ Elongation $\square$ Area reduction
Fig. 3. Mechanical properties of the as-rolled wires R1, R2 and $\mathrm{R} 3$, and the patented wire R4 determined by tensile tests

As already found and proven during prior examinations on samples of the medium carbon steel C45 and also eutectoid steel C80 in laboratory rolling experiments [5], the mean squared microstrain in the crystallographic direction $\langle 100\rangle, \varepsilon_{100}^{2}$, is a suitable parameter to characterize the materials properties means of FMA. The microstrain $\varepsilon_{100}^{2}$ and its anisotropy $q$ can be determined from the broadening of the diffraction peak (described by the FMA parameters $w$ and the shape parameter $m$ ) and are directly proportional to the density of microstructural defects causing this microstrain. In case of an as-rolled lamellar pearlitic structure those defects mainly refer to dislocations located at the ferrite/cementite interfaces [3, 4]. These so call misfit dislocations occur due to a lattice misfit between ferrite and cementite within the pearlite lamellar structure (as can be observed in transmission electron micrographs, see Figure 4) and were found to contribute the biggest part of the microstrains. Therefore it can be assumed that the finer the pearlite structure the higher will be the microstrains measured by FMA and a good-quality proportional correlation of $\varepsilon^{2}{ }_{100}$ and lamellar density, which was calculated as the reciprocal mean apparent interlamellar distance $I L D$ determined from SEM pictures could be observed. As widely known, e. g. [7], the pearlite lamellar density also correlates with the strength of fully pealitic steels. This relationship was applied to the $\mathrm{C} 80$ wire samples examined during this study and a reliable proportional correlation was found to be valid between lamellar density, tensile (and also yield) strength and the mean squared microstrain determined by FMA. Figure 5 shows the dependence between $\varepsilon_{100}^{2}$ and UTS of the industrial rolled pearlitic wires R1 to R4 compared to the correlations found for C45 and C80 samples during prior investigations in laboratory scale [5]. The offset of approx. $280 \mathrm{MPa}$ observed between the strength levels of both steel grades can be ascribed mainly to the different carbon contents [8] of $0.46 \%$ and $0.81 \%$, respectively, and obviously does not affect the slope of the linear dependence.

Additional microstructure characteristics can be calculated from the FMA diffraction lines of the as rolled wire samples. For example, the lattice parameters $a_{h k l}$ for the different crystallographic directions and their anisotropy can be determined from the shift of the diffraction lines related to the diffraction angle $2 \theta$ and are characterizing the $1^{\text {st }}$ kind residual stress condition of the examined samples. Furthermore, the analysis of the integrated intensities measured for the different $h k l$ by means of the FMA may be used to get information about preferred crystallographic orientations (texture) of the wire samples. According to [9] a texture index may be calculated from the $I_{h k l}$ and used as an indicator for the degree of preferred orientation. 


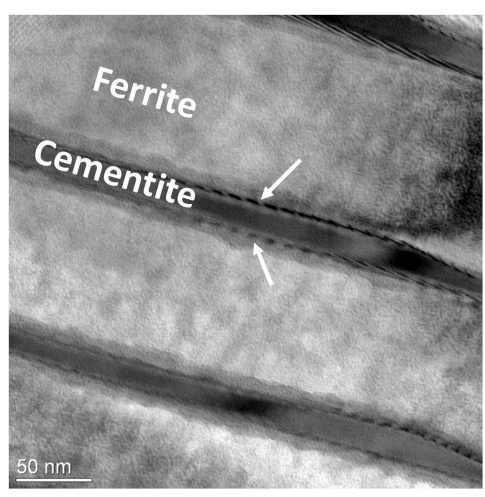

Fig. 4. TEM micrograph of pearlite. The misfit dislocations are visible by contrast (see arrows)

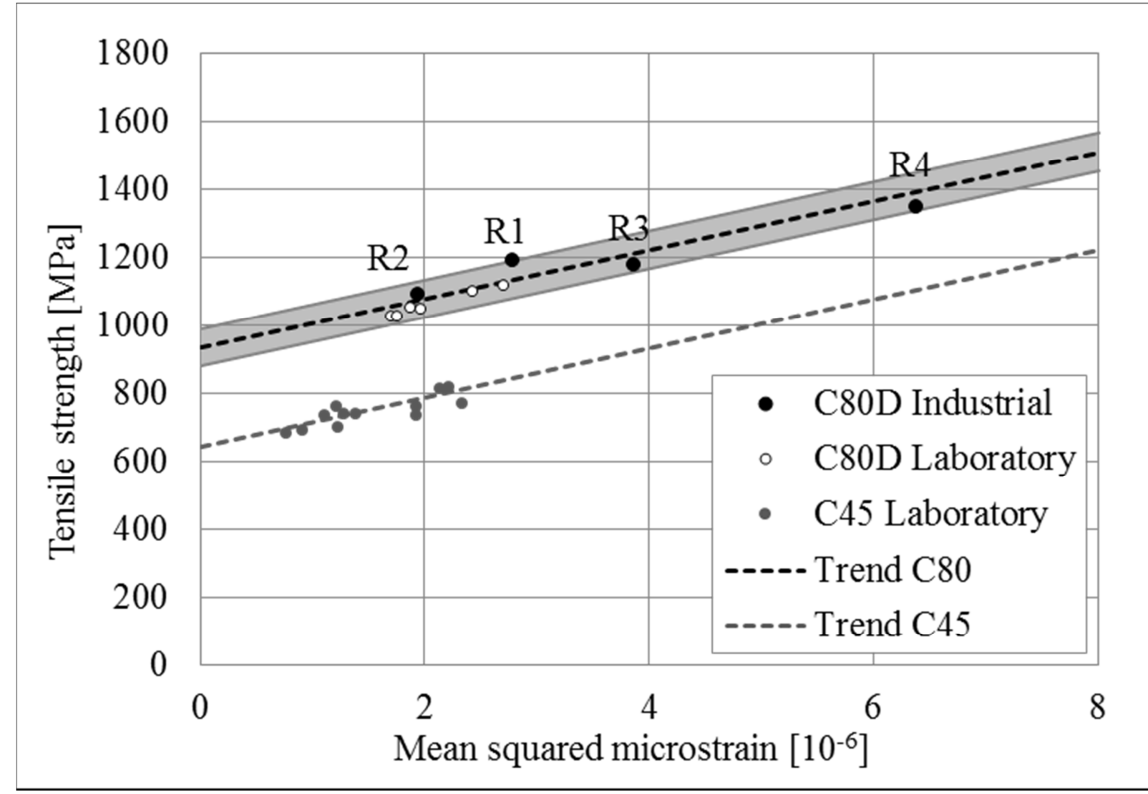

Fig. 5. Relationship between FMA determined microstrain and the ultimate tensile strength of the as-rolled / as-patented C80 samples compared to the tendencies observed for C45 and C80 samples during laboratory examinations

The lattice parameter in the $\langle 100\rangle$ direction $a_{100}$ was determined from the FMA measurements and found to only slightly deviate from the intrinsic value of $(2.8665 \pm 0.0002) \AA$ given by [10]. The anisotropic part of the lattice parameter, which is proportional to the residual stress, was near zero in all as-rolled samples as well as in the patented one indicating an almost residual stress-free condition (see Figure 6). The highest (positive) aberration was observed for the stelmor cooled sample R1, indicating low tensile residual stresses which can be explained by the more rapid (air) cooling in the temperature range of $\gamma / \alpha$-phase transformation during the stelmor cooling procedure after hot deformation. A preferred crystallographic orientation described by the ratio of the diffraction intensities $I_{110} / I_{200}$ determined according to [9], was not observed. The values obtained from the FMA and confirmed by pole figure measurements yielded approx. 1.0 indicating a random orientation for all samples R1 to R4.

Fig. 6. Lattice parameter $a_{100}$ of $\alpha$-ferrite and its anisotropy in as-rolled (R1 to R3) or as-patented (R4) condition determined by FMA

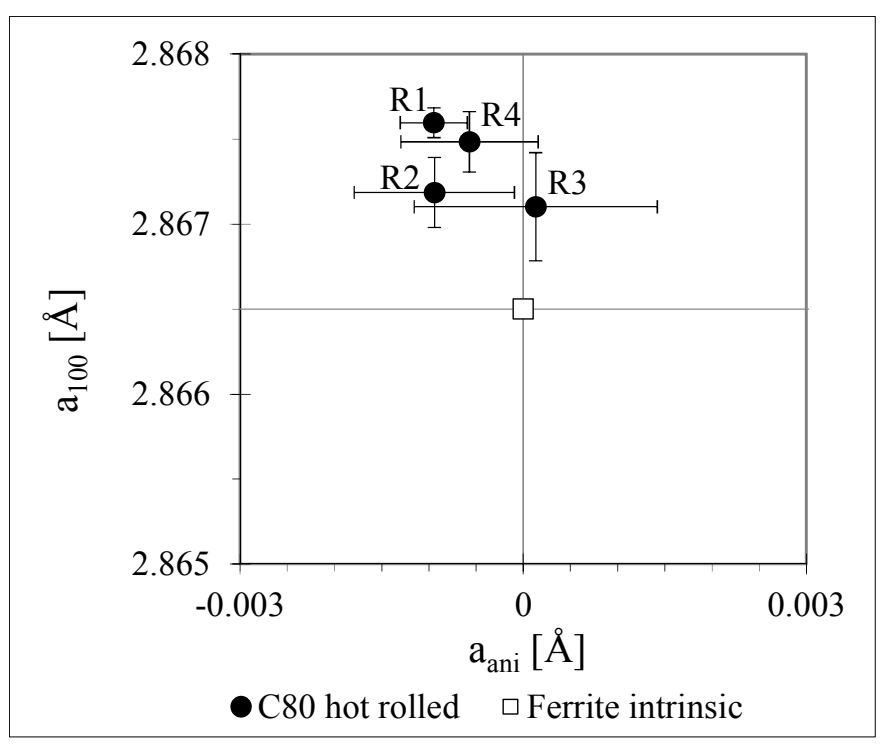

FMA after wire drawing. During wire drawing, the strengthening mechanism switches from microstructure refinement effects to strain hardening effect mainly responsible for the materials mechanical properties development. Prior investigations on a medium carbon steel C45 after drawing revealed a clear correlation between the $1^{\text {st }}$ kind residual stress state and the steel's strength 
after drawing. The lattice plane (111) and the corresponding lattice parameter $a_{111}$ determinable by FMA was identified as a suitable parameter to describe the strengthening effects during cold deformation and for tensile properties estimation from FMA measurements [5]. The model behind this correlation was based on the assumption that the deformation mainly takes place in the softer ferrite phase. The less ductile cementite phase leads to a compression of the ferrite perpendicular to the wire drawing axis after unloading and yields a reduction of the lattice parameter in the $\langle 111\rangle$ direction comparable to the Bauschinger effect. However, the investigations on the eutectoid steel C80 after industrial drawing reported about in this study showed only limited transferability of this approach developed for C45 steel. Due to the lack of primary ferrite and, hence, the lower total ferrite fraction of the fully pearlitic C80 steel the correlation was found only to work for true strains of $\varphi<1.0$, moreover, with a weaker reliability compared to C45 steel.

Therefore, a new model had to be developed for the application of FMA on as-drawn samples. This new approach was based on the known relationship between the macroscopic orientation of cementite lamellas and the crystallographic texture of the ferrite phase [11, 12]. To characterize the pearlite lamellar alignment during progressive wire drawing the mean disorientation angle $\beta$ of the cementite lamellas referring to the drawing direction was determined from SEM pictures by means of a newly developed evaluation method. This procedure can also be used for apparent ILD measurements. A detailed report about this method will be given elsewhere and a paper is currently in preparation.

Within the frame of the present work, an approach has been developed to correlate the mean pearlite disorientation angle $\beta$ with the texture characteristics evolving in the drawing direction within the wire during progressive drawing. The intensity ratio $I_{110} / I_{200}$ determined by the FMA has been detected to be a suitable correlation parameter. Because the lattice planes (110) and (200) enclose angles of either $45^{\circ}$ or $90^{\circ}$ their intensities react very sensitive to the fibre texture developing during progressive wire drawing. However, the texture analysis by evaluation of the integrated intensities of the XRD reflections in a particular direction is rather known to be suitable for qualitative estimation of preferred orientation than for quantitative predictions [13]. Therefore, additional pole figure measurements have been applied to verify the FMA-based results within the frame of this work.

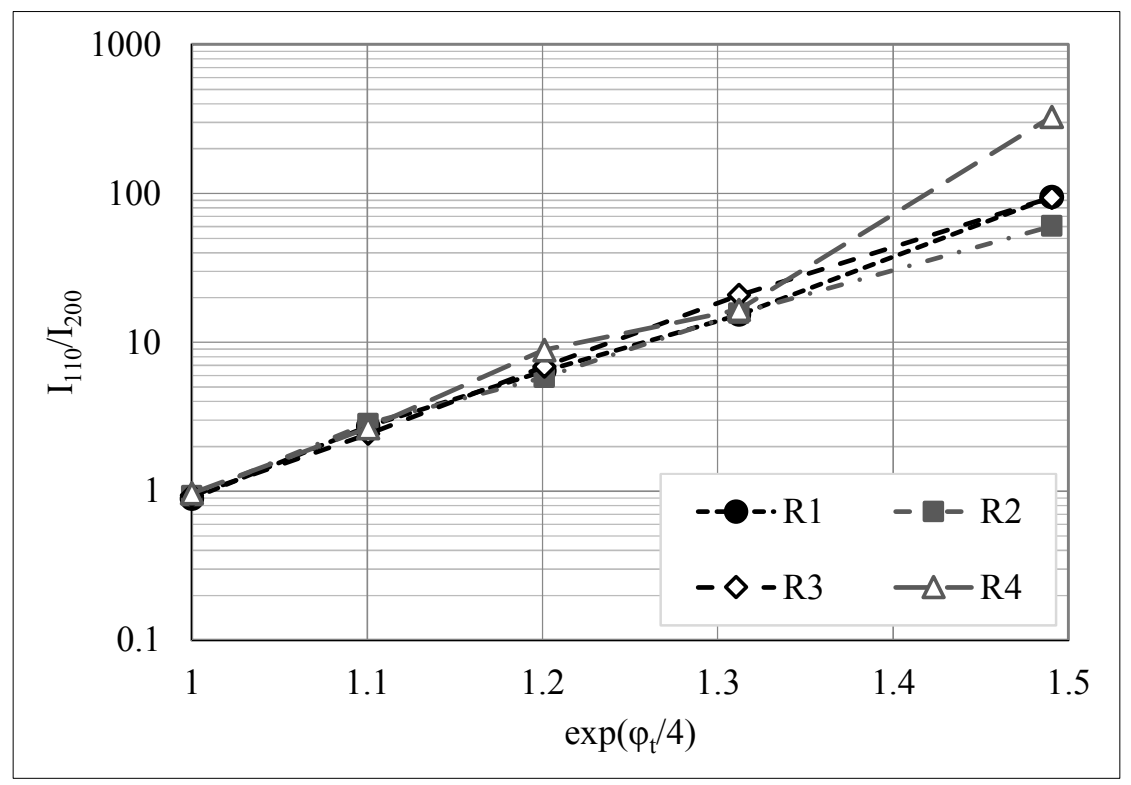

Fig. 7. Experimentally based dependence of the crystallographic texture perpendicular to the drawing direction from the total true strain applied during wire drawing

The diagram in Figure 7 shows the relationship between the texture evolution in drawing direction and the applied cumulative true strain $\varphi_{t}$ during industrial wire drawing process for the samples R1 to R4. The degree of crystallographic orientation expressed by the ratio $I_{110} / I_{200}$ was found to exhibit a direct proportionality to $\exp \left(\varphi_{t} / 4\right)$. According to the relationship introduced by [14] the strengthening of the C80 samples R1 to R4 during wire drawing could be proven to also correlate with the total plastic strains applied to the samples in our experiments. Therefore, a semi- 
logarithmic relationship between the intensity ratio $I_{110} / I_{200}$ and the ultimate tensile strength UST could be established for the examined samples (see Figure 8). Thereby, the initial pearlite structure developed after hot rolling and/or heat treatment prior to wire drawing was found to be substantial. It accounts for a significant basic information for estimating the strength level (referring to the offset of the semi-logarithmic dependency) during wire drawing and for the estimation of the UTS of a certain sample from the FMA determined microstructure parameters.

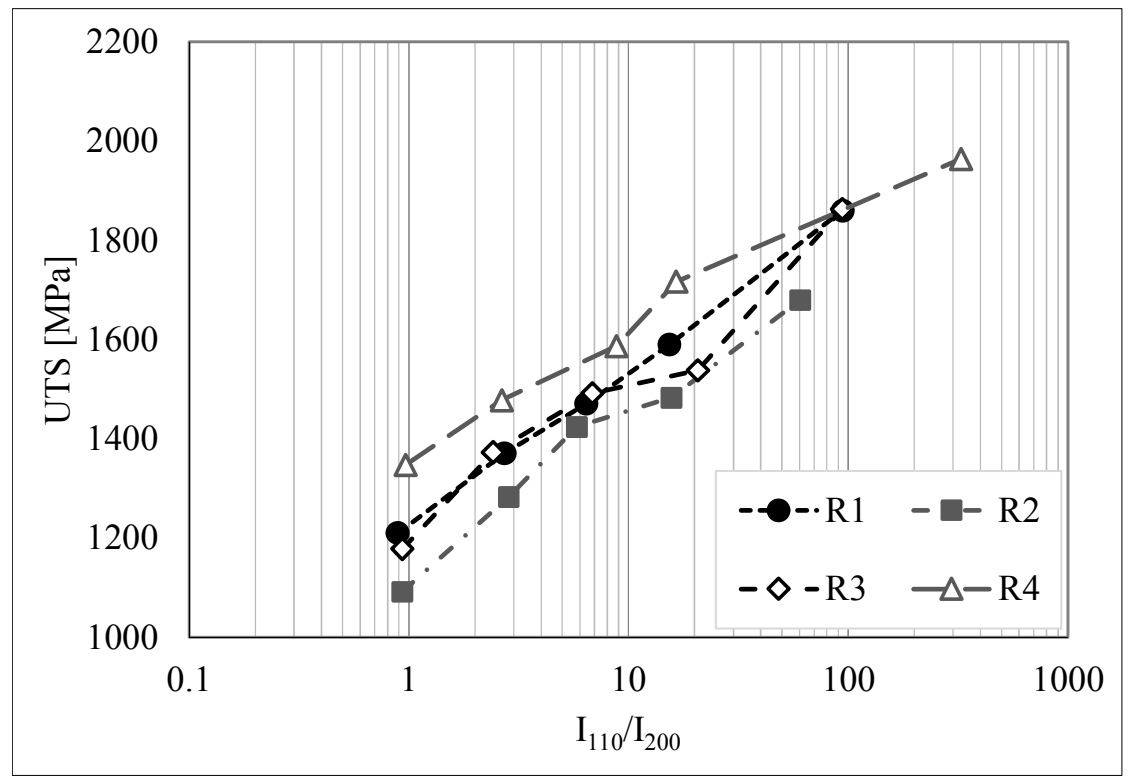

Fig. 8. Examined dependence between the ultimate tensile strength UTS and the crystallographic texture perpendicular to the drawing direction after wire drawing determined by means of FMA

\section{Summary and Conclusions}

Within the frame of this work, a newly developed method for the fast analysis of multicomponent steel's specific microstructure characteristics after hot and cold deformation has been applied and tested for industrial pearlitic spring steel wire production process. The method is based on X-ray diffraction measurements of distinct $h k l$ reflections for the bcc ferrite lattice structure. By parametrization of the diffraction lines and by calculation of typical microstructure parameters, lattice deformations and internal stresses and stress fields according to the crystallographic direction were investigated and quantified.

The mean squared microstrain in the $\langle 100\rangle$ direction $\varepsilon^{2} 100$ was identified as an important value for characterizing as-rolled or heat treated randomly oriented lamellar pearlite structures and for estimating their macroscopic tensile strength properties. The linear dependence discovered during prior investigations on a medium carbon steel grade $\mathrm{C} 45$ was generally confirmed. The strength of the as-rolled or as-patented steel grades can be estimated from FMA with a precision of $\pm 50 \mathrm{MPa}$ (cp. Figure 5). Additional microstructure information (about residual stresses and preferred orientation), that may also affect the steel's strength properties, can be determined by the FMA, too.

For the cold drawn wire samples the strengthening during progressive cold deformed was found to correlate appropriately with the texture index $I_{110} / I_{200}$ determined from FMA. A semi-logarithmic dependence between the UTS and the intensity ratio was observed for the examined samples. For UTS estimation based on this correlation the initial strength of the wire prior to cold drawing is required to define the strength level. Additional information provided by the FMA concerning the $1^{\text {st }}$ kind residual stress state can be used to classify the material condition with respect to the technological history.

The FMA is suitable for extending the possibilities of process monitoring and quality assurance during high-strength pearlitic steel wire production. The major advantages of the method are the time saving procedure, compared to standard metallographic examinations, due to uncomplicated sample preparation and a fast and automated measurement and evaluation as well as the high information content. Nevertheless, it has to be considered that the microstructure models and correlations are partly material specific. Therefore, a set of reference data will be necessary for each 
steel grade based on the technological background. So far only two non-alloyed steel grades have been examined in detail and further work will have to be invested to tap a wider spectrum of steel grades and alloys.

The FMA measurements gain access to multiple microstructure information and were found to be very sensitive also to slight microstructure deviations. By refinement and further development of the basic microstructure models a higher precision and reliability of the mechanical properties prediction will be achievable and specific measuring effects will be attributable to certain technological and/or microstructural causes.

Following on the results presented in this paper, the FMA has already been tested on components fabricated from as-drawn pearlitic steel wires, too. Coiled compression springs have been chosen as a typical example and a possible application. The insights gained during these investigations are promising, even though the clarification of the relevant technology based microstructure/property relations will take even more effort to enable concrete conclusions to be drawn on the component properties.

\section{Acknowledgment}

The authors wish to thank the German Scientific Council (DFG) for supporting their work within the scope of technology transfer. Special thank is addressed to our partners in industry supporting us by putting their production resources into the service of science.

\section{References}

[1] W. Lehnert, Draht 48 (1997) No. 4, 44-48.

[2] D. Šimek, D. Rafaja, M. Motylenko, V. Klemm, G. Schreiber, A. Brethfeld, G. Lehmann, Steel Research Int. 79 (2008) 800-806.

[3] A. Oswald, R. Schmidtchen, D. Šimek, M. Motylenko, G. Lehmann, D. Rafaja, Conference Proceedings - MEFORM 2013, Altenberg (2013) 327-343.

[4] D. Šimek, M. Motylenko, A. Oswald, R. Schmidtchen, G. Lehmann, D. Rafaja, Zeitschrift für Kristallografie Suppl. 30 (2013) 29-30.

[5] D. Šimek, A. Oswald, R. Schmidtchen, M. Motylenko, G. Lehmann, D. Rafaja, Steel Research Int. 85 (2014) 1369-1378.

[6] M. M. Hall Jnr, V. G. Veeraraghavan, H. Rubin, P. G. Winchell, Journal of Appl. Crystallography 10 (1977) 66-68.

[7] K.K. Ray, D. Mondal, Acta Met. Mat. 39 (1991) 2201-2208.

[8] T. Gladman, I. D. McIvor, F. B. Pickering, Journal of the Iron and Steel Inst. 210 (1972) 916930.

[9] G. B. Harris, Philos. Mag. 43 (1952) 113-123.

[10] FIZ Karlsruhe, ICSD - Inorganic Crystal Structure Database, CD-ROM-Ed., V 1.4.1 (2006).

[11] M. Zelin, Acta Materialia 50 (2002) 4431-4447.

[12] J. Toribio, B. González, J.-C. Matos, Materials Transactions 55 (2014) 93-98.

[13] J. Ricotea, D. Chateigner, Journal of Appl. Crystallography 37 (2004) 91-95.

[14] J. D. Embury, R. M. Fisher, Acta Metallurgica 14 (1966) 147-159. 